\title{
Existence Is Evidence of Immortality
}

\author{
by Michael Huemer
}

Abstract: Time may be infinite in both directions. If it is, then, if persons could live at most once in all of time, the probability that you would be alive now would be zero. Since you are alive now, with certainty, either the past is finite, or persons can live more than once.

\section{Overview}

Do persons continue to exist after the destruction of their bodies? Many believe so. This might occur either because we have immaterial souls that persist in another, non-physical realm; or because our bodies will be somehow reanimated after we die; or because we will live on in new bodies in the physical realm. ${ }^{1}$ I shall suggest herein that the third alternative, "reincarnation," is surprisingly plausible. More specifically, I shall argue (i) that your present existence constitutes significant evidence that you will be reincarnated, and (ii) that if the history of the universe is infinite, then you will be reincarnated.

My argument is entirely secular and philosophical. The basic line of thought is something like this. The universe has an infinite future. Given unlimited time, every qualitative state that has ever occurred will occur again, infinitely many times. This includes the qualitative states that in fact brought about your current life. A sufficiently precise repetition of the right conditions will qualify as literally creating another incarnation of you. Some theories about the nature of persons rule this out; however, these theories also imply that, given an infinite past, your present existence is a probability-zero event. Hence, your present existence is evidence against such theories of persons. Given an infinite past, it is conclusive evidence.

In what follows, I elaborate the reasoning more precisely and address objections to the idea of reincarnation, including that the correct theory of personal identity rules out reincarnation, that the notion of reincarnation requires a false doctrine of mind/body dualism, and that the heat death of the universe will prevent our living again.

\section{The Infinitude of Time}

\subsection{Intuitive Support for an Infinite Past}

It is widely accepted that the future of the universe is infinite. I shall therefore take that for granted in what follows. It is, however, controversial whether the past is infinite. Here, I shall contend that it is at least plausible that the universe has an infinite history.

My main reason for this is intuitive: the idea of a beginning of time seems to me metaphysically impossible in a manner similar to that of an edge of space. For any location in space, it makes sense to ask what is, for example, one meter to the left of it (even if the answer is that nothing is there). If there were an edge of space, what would happen if you approached it and tried to gaze past the edge? When we try to imagine this, we find ourselves trying to imagine a place where there is no space, which is of course impossible. The idea of a bounded

\footnotetext{
1 The second alternative is endorsed by, e.g., van Inwagen (1978). The third is defended by, e.g., Almeder (1992).
} 
space is perfectly mathematically consistent, it simply does not seem that Space, that is, all of space, could have this structure.

Similarly, for any moment in time, it makes sense to ask what happened, say, one minute before (even if the answer is that nothing happened). If there were a beginning of time, it would have to somehow not make sense to ask what happened before that time. When we try to conceive of the beginning, we find ourselves trying to imagine time coming into existence. But time could not have come into existence, because a thing's coming into existence implies a time when the thing does not exist, followed by a time when the thing exists. Of course there could not have been a time when time did not exist. Sometimes it is suggested that God, existing outside time, created time. ${ }^{2}$ But even God could not do this, because any act of creation - or any other action or event - presupposes a time at which the act or event may take place. One can identify, of course, a consistent mathematical structure containing a first time; it just does not seem that Time, that is, all of time, could have this structure.

\subsection{Philosophical Objections to an Infinite Past}

Some philosophers believe that it is the notion of an infinite past that is metaphysically impossible, either because there cannot be an "actual infinity," or because an infinite series can never be completed by proceeding through the series one step at a time. ${ }^{3}$

Among other problems, these sorts of arguments run afoul of Zeno's paradoxes. Suppose I drop a ball. To reach the ground, the ball must first go half the distance, then half the remaining distance, then half the remaining distance again, and so on. The series is infinite, and the stages must be completed one at a time, in order. Nor are these steps merely potential: each of these steps must actually be completed before the ball reaches the ground; if any of them is merely capable of being completed but not in fact completed, then the ball has not reached the ground. Pace Zeno, however, dropped objects do in fact reach the ground.

The series just described has a first step but no last step. We can also have a series with no first step: Consider again the ball that is to fall from my hand to the ground. Before reaching the ground, it must reach the halfway mark; but before it does this, it must reach the one quarter mark; before that, the one eighth mark; and so on. This series of events is infinite, and (like the series of past events in the history of the universe) it has no step that comes first in time. Nevertheless, objects complete such series every time they move. ${ }^{4}$

The philosophical objections to an infinite past are therefore misguided.

\subsection{Time in Modern Cosmology}

The standard version of Big Bang cosmology has it that spacetime itself began with the Big Bang singularity, about 14 billion years ago. This is the strongest reason for thinking time may be finite in the past direction, though, again, still infinite in the future direction. ${ }^{5}$

However, the finitude of the past remains a matter of debate, as some contemporary cosmological models incorporate infinite past time. Most of these are cyclic models, in which the universe goes through an infinite series of big bangs, each followed by a phase of

\footnotetext{
${ }^{2}$ Craig 2001, ch. 6.

${ }^{3}$ Craig 1991. For an extended rebuttal to such arguments, see Huemer 2016, chs. 4-5.

${ }^{4}$ For discussion, see Huemer 2016, pp. 41-4, 185-6.

5 See Davies 1984, ch. 2; Mithani and Vilenkin 2012.
} 
expansion, followed by a contraction or other form of resetting to the initial state. This includes the Steinhardt-Turok model, the Baum-Frampton model, loop quantum cosmology, and Penrose's conformal cyclic cosmology. ${ }^{6}$ Other theories postulate an infinite multiverse that periodically spawns daughter universes, where each daughter universe has a finite age while the multiverse is of infinite age. ${ }^{7}$

One problem with the standard (finite-past) Big Bang theory is that the theory requires the universe to have simply started out, for no reason, in an extraordinarily improbable state, one with extremely low entropy. It has been calculated that the probability of the initial state being as the theory takes it to be (if, so to speak, an initial state were chosen randomly) is less than 1 in $10^{10^{124}} \cdot 8$

You might think that, despite its improbability, it is permissible to postulate the very lowentropy initial state for the universe, if this is required to explain what we observe. But it is not required, for here is an alternative hypothesis: Perhaps, rather than 14 billion years ago, the universe began in 1950, with everything in the state that we in fact believe it to have been in then - including human brains configured so as to have false memories of an earlier past, including dinosaur bones buried in the ground just as if dinosaurs had lived millions of years ago, and so on. This hypothesis is many orders of magnitude more likely than the standard Big Bang theory - that is, if you consider all the physically possible configurations for all the particles in the universe, a larger proportion of these configurations have the universe being as we think it actually was in 1950 than have it being as we think it was at the start of the Big Bang. ${ }^{9}$ So if you are postulating a starting state with no explanation, you might as well postulate the 1950 starting state.

You might object to my probabilistic claims. Perhaps you think, for one reason or another, that initial probabilities cannot be assigned to possible initial states of the universe. In that case, the 1950 initial state cannot be said to be more likely than the Big Bang initial state. But neither can it be deemed less likely. Meanwhile, the two theories are otherwise parallel: both explain all observations made since 1950 (which, if you were born since 1950, includes all of your observations), and both do so by postulating a beginning of time, with an uncaused and unexplained initial state of the universe at that time. One cannot object to the improbability or inexplicability of the 1950 initial state, without impugning the Big Bang theory's postulated initial state. Thus, if the standard Big Bang theory is reasonable, then the 1950 theory is also reasonable. But I take it that the 1950 theory is not reasonable.

I conclude that it is at least plausible that the universe has an infinite past, in addition to an infinite future. It is therefore interesting to consider what follows if the past is infinite.

\footnotetext{
${ }^{6}$ See Steinhardt and Turok 2005; Baum and Frampton 2007; Ashtekar 2009; Penrose 2010.

${ }^{7}$ See Susskind 2006; Carroll 2010.

${ }^{8}$ Penrose 2010, p. 127.

${ }^{9}$ This is the meaning of the statement that the universe's entropy greatly increased from the time of the Big Bang to 1950. Cf. Chen's (2017, pp. 16-17) hypothesis that we live in a low-entropy bubble resulting from a thermodynamic fluctuation 200 years ago. This, similarly, is much more probable than the Big Bang theory, on the standard probability measure used in thermodynamics.
} 


\section{Infinitude Yields Eternal Recurrence}

According to the doctrine of Eternal Recurrence, embraced by Seneca, Nietzsche, and others, everything that occurs will repeat itself in the future, infinitely many times. ${ }^{10}$ Notice that this is not a priori guaranteed to be true, even if the future is infinite. To see this, imagine a universe consisting of a single pair of particles. At $t_{0}$, the particles are one meter apart and heading away from each other (at more than escape velocity). At all later times, the particles will be farther apart; they will never return to their initial configuration.

Nevertheless, eternal recurrence holds in a wide and interesting class of cases, probably including the case of our actual universe. The Poincaré Recurrence Theorem states that a physical system limited to a bounded region of phase space will (with probability 1) return arbitrarily close to its initial state given sufficient time. Given infinite time, it will repeat the initial state, to any desired degree of precision, infinitely many times. ${ }^{11}$ Here, the "initial state" simply refers to the state at some arbitrary moment that we choose to start considering the system.

The theorem applies only to systems confined to a bounded region of phase space. The phase space of a physical system is an abstract, mathematical space containing all the possible configurations of the system. At any given time, the system occupies a point in this space, determined by the values of all its physical variables. As it evolves over time, it "moves" through phase space - that is, it changes the values of its physical parameters. If it remains within a limited region in this abstract space (as must happen, for example, if all its particles are confined to a finite region of physical space with finite energy), then the recurrence theorem applies. The theorem does not apply to the two-particle system mentioned above, since that system is not confined to a bounded region.

Does the Poincaré Recurrence Theorem apply to the universe as a whole? It may not, for the universe may continue expanding indefinitely, in which case its phase space is unbounded. But if our universe has an infinite past as suggested in section 2 above, then it is probably cyclical, meaning that it does not expand infinitely but goes through cycles of expansion and contraction. In that case, it will repeat its present state, to any desired degree of precision, at some future time. If instead we are part of an infinite multiverse that periodically spawns new daughter universes, then, although our universe may never return to its current state, other daughter universes will approximate to our universe's current state, with any desired degree of precision. Note also that the argument for reincarnation only requires there to be a recurrence of a person sufficiently similar to you; the entire surrounding universe need not be the same.

I conclude that Eternal Recurrence is likely, especially so if the past is infinite.

10 Seneca 2010, letter 36. Nietzsche (2001, section 341) first introduces eternal recurrence as a thought experiment; later (2003, pp. 23-4, 112, 118), he claims that physics guarantees eternal recurrence.

${ }^{11}$ For a helpful exposition, see Albert 2000, pp. 73-4. The traditional theorem applies in the context of classical physics. For a generalization covering both quantum and classical contexts, see Wallace 2015. 


\section{Recurrence Yields Reincarnation}

\subsection{The Bayesian Conception of Evidence}

Before explaining the evidence of immortality, I need to explain two general points about the nature of evidence. These are the Bayesian Conception of Evidence, and the Indexicality of Evidence.

What I call the Bayesian Conception of Evidence is an idea motivated by probability theory, especially Bayes' Theorem. The idea is that evidence supports a hypothesis when that hypothesis renders the evidence more probable. That is, the evidence should be something that would be more likely if the hypothesis in question were true than if it were not. In mathematical terms:

Bayesian Conception of Evidence: $\mathrm{E}$ supports $\mathrm{H}$ if $\mathrm{P}(\mathrm{E} \mid \mathrm{H})>\mathrm{P}(\mathrm{E} \mid \sim \mathrm{H})$

This is subject to the qualification that the initial probabilities of $\mathrm{H}$ and $\sim \mathrm{H}$ must each be nonzero (otherwise, neither can be supported). Furthermore, the degree to which evidence supports a hypothesis is determined by the likelihood ratio, $\mathrm{P}(\mathrm{E} \mid \mathrm{H}) / \mathrm{P}(\mathrm{E} \mid \sim \mathrm{H})$ : the higher this ratio is, the more strongly $\mathrm{E}$ supports $\mathrm{H}$.

Why hold this view? Because it is a theorem of probability that whenever $\mathrm{P}(\mathrm{E} \mid \mathrm{H})>$ $\mathrm{P}(\mathrm{E} \mid \sim \mathrm{H})$ (and $\mathrm{P}(\mathrm{H})$ and $\mathrm{P}(\sim \mathrm{H})$ are nonzero), then $\mathrm{P}(\mathrm{H} \mid \mathrm{E})>\mathrm{P}(\mathrm{H})$, which means that the truth of $\mathrm{E}$ makes it more likely that $\mathrm{H}$ is true. For any given value of $\mathrm{P}(\mathrm{H})$, it can be shown that as the likelihood ratio $(\mathrm{P}(\mathrm{E} \mid \mathrm{H}) / \mathrm{P}(\mathrm{E} \mid \sim \mathrm{H})$ ) increases, the ratio $\mathrm{P}(\mathrm{H} \mid \mathrm{E}) / \mathrm{P}(\mathrm{H})$ increases monotonically.

I have so far taken no stand on what propositions should be considered to correctly state our evidence. I have assumed only that there are some propositions that state our evidence, and that, whatever they are, if they render some theory more likely to be true, then we have evidence for that theory.

\subsection{The Indexicality of Evidence}

Sometimes, our evidence is indexical - that is, it can only be correctly characterized using an indexical expression such as "I" or "now", rather than using purely qualitative terms. To illustrate, imagine that you flip a coin ten times. To your surprise, you observe it come up heads all ten times. Question: Do you now have evidence that many coins are flipped at least ten times each, somewhere in the universe, by someone?

I assume the answer is obviously no. But suppose we were to describe the evidence, nonindexically, as that a coin comes up heads ten times in a row. The probability that a coin comes up heads ten times in a row is higher if many coins are repeatedly flipped, than if not many coins are repeatedly flipped. For example, to a first approximation, it is twice as likely that at least one coin comes up heads ten times in a row if there are two coins being repeatedly flipped than if there is only one. It is even more likely if there are a thousand coins, and almost certain if there are millions of coins. Applying the Bayesian conception of evidence, therefore, we might claim that the evidence supports that there are many coins that are flipped, somewhere in the universe. 
The fallacy is straightforwardly diagnosed: the evidence one acquires in the scenario is not merely that some coin comes up heads ten times in a row. The evidence one acquires is that this specific coin comes up heads ten times in a row. Flippings of other coins, however many there may be, have no effect on the probability of this coin's coming up heads ten times. Therefore, the observation of this coin's behavior is not evidence either for or against the existence of any such other coin flips. ${ }^{12}$

A similar point can be made about time: if we characterize the evidence merely as that this coin comes up heads ten times in a row (at some time), then we can derive the perverse conclusion that your observation of the ten heads is evidence that this coin was flipped many times in the past or will be flipped many times in the future. The correct characterization of the evidence, for purposes of applying the Bayesian Conception of Evidence, must be "this coin came up heads ten times in a row just now." Any past or future flips of the coin would have no effect on its probability of having come up heads ten times just now; hence, the evidence does not either support or undermine the hypothesis of any earlier or later flips.

There are ways of eliminating the specific indexical terms I have used in this example. For instance, one could eliminate the expression "this coin" in favor of "the coin most recently observed by me," and the expression "just now" in favor of "the time immediately preceding the thinking of this sentence." But these ways just substitute other indexical expressions. Any correct description of the evidence, for purposes of deciding what hypotheses are supported by the evidence, must employ some indexical expression(s). Not all of our evidence need be indexical in this sense, but at least some of it is.

Why does the indexical statement, "This coin came up heads just now," beat the purely qualitative statement, "A coin came up heads at a time," as a report of one's evidence? Plausibly, this is accounted for by the general principle that the most informative, relevant statement available must be used when we update our beliefs in the light of evidence. ${ }^{13}$ When you observe the coin, you indeed learn that a coin came up heads at some time. But you also learn that this coin came up heads at that specific time. The latter statement is more informative (logically stronger) than the former, so the latter must be used rather than the former, whenever this would make a difference to the calculated probabilities of hypotheses in the light of the evidence.

An analogous point applies to the evidence you have in virtue of your present conscious experiences. It would be a mistake to characterize this evidence as that a being with such-and-such experiences and characteristics exists at some time. Rather, when you are aware of your own experiences, you have, as evidence, that you are having such-and-such experiences now. The point applies also to your awareness of your existence as a living, conscious being. You do not merely have as evidence that some conscious being, satisfying some purely qualitative description, lives at some time. You have as evidence that you are alive now. This point will be important in the reasoning to follow.

12 For elaboration, see Hacking 1987; Huemer 2018, 227-9.

13 See Hempel 1960. 


\subsection{Theories of Persons}

As discussed in section 3, it is likely that the universe in the future will contain persons who will be very similar to you - with any desired degree of similarity, in any desired respects. This would happen simply as a result of blind rearrangement of particles according to physical law. Let us call these persons your "Poincaré clones."

Are your Poincaré clones incarnations of you? Many would say that a Poincaré clone is merely another individual very much like you, rather than literally you. When we speak of personal survival, we are interested in the existence of the very same token individual after the destruction of their body, not the appearance of another individual of the same type. Hence, many would say, eternal recurrence is irrelevant to personal survival. ${ }^{14}$

Whether a Poincaré clone of you is literally you depends upon the correct theory of persons. At least some non-absurd theories of persons would allow your clones to be literally (different stages of) you. Call these "Permissive" theories of persons. Thus, perhaps there are certain repeatable characteristics such that, whenever there is a unique person having those characteristics, that person counts as you. These characteristics could include a certain type of brain configuration, certain mental capacities, certain character traits, and so on. They could include complex, disjunctive properties; dispositional properties; relational properties; or any other repeatable properties, taking "properties" in the broadest sense. Note: Despite the picturesque talk of Poincaré clones, a Permissive theory need not demand a high degree of similarity between your current and future incarnations; a theory counts as Permissive just as long as the conditions for being you are held to be repeatable.

On the other hand, some theories of persons would rule out counting your Poincaré clones as you. Call these "Restrictive" theories of persons. For instance, perhaps there is a spatiotemporal continuity requirement on personal identity, so that $\mathrm{A}$ and $\mathrm{B}$ count as stages of the same person only if there is a spatiotemporally continuous series of person-stages connecting A to B. Or perhaps the incarnation of a particular individual metaphysically requires a particular token event as cause. Both of these views would rule out your being reincarnated; at most, according to these views, the future might contain a token-distinct person who will be qualitatively indistinguishable from you. Many other Restrictive theories are possible.

For the reasoning to follow, it is not necessary that we initially accept a Permissive view of persons. It is only necessary that we be less than $100 \%$ certain of a Restrictive view. That is, I assume that there is a nonzero probability that some Permissive theory is true.

\subsection{Existence Confirms Reincarnation}

Your present existence as a living, conscious being is evidence for a Permissive view of persons, and hence for actual reincarnation. From the Indexicality of Evidence (section 4.2), the proper characterization of the evidence is that you are alive now; your evidence is not, for example, merely that some person having such-and-such qualitative characteristics lives at some time. From the Bayesian Conception of Evidence (section 4.1), this counts as evidence for a Permissive view of persons, provided that the probability of your being alive now given

\footnotetext{
14 van Inwagen 1978, pp. 116-18; Jenkins 2012, p. 210.
} 
a Permissive view of persons is greater than the probability of your being alive now given a Restrictive view of persons, that is:

$$
\mathrm{P}(\mathrm{L} \mid \mathrm{H})>\mathrm{P}(\mathrm{L} \mid \sim \mathrm{H})
$$

where $\mathrm{L}=$ [You live now], and $\mathrm{H}=$ [Some Permissive view of persons is correct]. And this probabilistic condition in fact obtains. You would be more likely to be living now if persons could live many times than if persons could only live once in the history of the universe. Whether we take a Permissive or Restrictive theory of personal identity has no effect on the probability of there being a person qualitatively like you existing at the present time - but a Permissive theory makes it more likely that such a person would be you. In fact, as I shall presently show, given an infinite past and a Restrictive theory of persons, the probability of your being alive now would be zero.

We start with a general lemma about unrepeatable events. Let $E$ be any event that may occur at a particular time. Suppose we are given the following:

i. E can occur at at most one time in the history of the universe.

ii. The history of the universe is infinite.

iii. E is initially no more likely to occur at any given time than at any earlier time. ${ }^{15}$

Conditional on just these assumptions, the probability of E occurring now or in the recent past is zero.

Why zero? If it occurs at all, E must occur within some particular century, either the present one or an earlier one. (By stipulation, count the last 100 years as "the present century". A century is an arbitrarily chosen time interval; any nonzero time interval will work for the argument.) Suppose the prior probability that E would occur in the present century is said to be $1 \%$. Given assumption (iii) above, the prior probability of E's occurring in the previous century must be at least $1 \%$, and in the century before that also at least $1 \%$, and so on. Given assumption (i), these probabilities are additive: the probability of E's occurring in the previous two centuries would be at least $2 \%$, in the previous three centuries at least $3 \%$, and so on. That means that the prior probability of E's occurring within, say, the past 200 centuries would be at least $200 \%$, which is absurd. The same problem occurs if we instead choose a probability of $0.1 \%$ (just consider the previous 2000 centuries), $0.01 \%$ (consider the past 20,000 centuries), and so on. Given an infinite past, the problem can be generated for any nonzero probability. The only way to avoid probabilities adding up to more than $100 \%$ is for the probability of E's occurring in the present century to be zero.

Here is a perhaps more intuitive version of the reasoning: $\mathrm{E}$ has either zero chance or a nonzero chance of occurring per century, if it hasn't happened previously. (a) Suppose E has

\footnotetext{
$15 \mathrm{I}$ assume only that $\mathrm{E}$ is not more likely to occur at later times (i.e., during later time intervals) than at earlier times (i.e., in earlier, equal-sized intervals). E might in fact be less likely to occur at later times than at earlier times, because its occurrence at an earlier time could prevent its later occurrence, but not vice versa. Analogy: suppose a coin is to be flipped repeatedly until the first time it comes up tails. The flip on which tails occurs is more likely to be earlier rather than later: It is $50 \%$ likely to be the first flip, $25 \%$ likely to be the second, $12.5 \%$ likely to be the third, and so on.
} 
a nonzero chance of occurring per century if it hasn't happened previously. Given an infinite number of centuries, then, it would certainly happen. If the past is infinite, then an infinite number of centuries have in fact occurred before the present century. So, with certainty, E would have happened before the present century. But according to assumption (i), E can happen at most once; thus, its having happened before the present century prevents it from happening in the present century. Therefore, E would certainly not happen in the present century. (b) Now suppose instead that E has zero chance of occurring per century if it hasn't happened previously. Then, with certainty, E does not happen in the present century. So, whichever assumption we make, we predict, with certainty, that E does not happen in the present century.

If a theory predicts, with probability 1 , that some event should not occur, and that event in fact is known to occur, then the theory is thereby conclusively refuted. As just discussed, the conjunction of (i), (ii), and (iii) predicts, with certainty, that E does not occur in the present century. If we know for certain that $\mathrm{E}$ has occurred in the present century, then we must reject the conjunction of (i)-(iii). If we are confident of (ii) and (iii), we should reject (i). If we have some initial credence in each of (i)-(iii) but are unsure of each, then we should lower our credence in all three.

Now substitute your being incarnated (that is, beginning a life as a conscious being) for E. According to Restrictive theories, (i) you can only live once in the history of the universe. As discussed above (section 2), it is plausible that (ii) the history of the universe is infinite. Finally, (iii) a priori, if you were to live in some century, it is no more likely that it would be the present century than that it would be any given previous century. On the Restrictive view, your living in any past century would have prevented you from living now. Since there are infinitely many past centuries, this should have happened long ago, unless the probability of your being born in any given century is zero. But in that case, the probability of your being alive now would be zero. Since you know for certain that you are alive now, you must reject, with full confidence, the conjunction of (i)-(iii). If you are fully confident of (ii) and (iii), you should reject (i). Even if you are not fully confident of (ii) and (iii), as long as you are less than fully confident of (i), you should lower your credence in (i) upon noticing that (i)-(iii) together predict your present non-existence. It does not matter what is the initial probability of a Permissive theory being correct, nor how likely you would be to be alive now given a Permissive theory, as long as these probabilities are nonzero. Since a Restrictive view of persons predicts zero probability of your existing now, it is certain that Restrictive views are false.

\subsection{An Analogy}

Here is an analogy to further illustrate the logic. Suppose there are two demons, Q1 and Q2, who periodically kidnap people. Q1's procedure is as follows: After kidnaping a victim, he flips a fair coin ten times within an hour. If it comes up tails at least once, he kills the victim. If it comes up heads all ten times, he releases the victim unharmed.

Q2 is even more diabolical. After kidnaping a victim, Q2 flips a fair coin an infinite number of times within one hour, using his demonic supernatural powers. If it ever comes up tails in this infinite series, he kills the victim. If it comes up heads on all flips, he releases the victim unharmed. 
Now suppose you have just been kidnaped by either Q1 or Q2; you can't tell which, and you assign a nonzero chance to each. You anxiously await your fate. One hour later, you find yourself being released unharmed. Given this experience, which demon kidnaped you?

The answer is Q1. It is initially unlikely that you would still be alive an hour after being kidnaped by Q1. But it is much less likely that you would still be alive after being kidnaped by Q2. In fact, though it is logically possible for the coin to come up heads infinitely many times in a row, the probability of this happening is zero. So you can infer, with probability 1 , that this did not happen, and therefore that it was not Q2 who kidnaped you.

The Bayesian calculation would go as follows, where $q_{1}$ is the proposition that you are kidnaped by Q1, $q_{2}$ the proposition that you are kidnaped by $\mathrm{Q} 2$, and $s$ the proposition that you survive:

$$
P\left(q_{1} \mid s\right)=\frac{P\left(q_{1}\right) \cdot P\left(s \mid q_{1}\right)}{P\left(q_{1}\right) \cdot P\left(s \mid q_{1}\right)+P\left(q_{2}\right) \cdot P\left(s \mid q_{2}\right)} .
$$

As given in the story, $\mathrm{P}\left(s \mid q_{1}\right)=1 / 1024$, while $\mathrm{P}\left(s \mid q_{2}\right)=0$. Plugging this into the above equation, as long as $\mathrm{P}\left(q_{1}\right)$ is nonzero, we get:

$$
P\left(q_{1} \mid s\right)=\frac{P\left(q_{1}\right) \cdot(1 / 1024)}{P\left(q_{1}\right) \cdot(1 / 1024)+P\left(q_{2}\right) \times 0}=\frac{P\left(q_{1}\right) \cdot(1 / 1024)}{P\left(q_{1}\right) \cdot(1 / 1024)}=1 .
$$

To make the analogy explicit: in the above scenario, the probability that you would be alive now if you had been kidnaped by Q2 is zero. Therefore, once you find that you are alive now, as long as there is a nonzero initial probability that it was Q1, you can infer with certainty that it was indeed Q1 who kidnaped you. Similarly, the probability that you would be alive now, if persons were unrepeatable (could not be reincarnated) and there were an infinite past, is zero. Therefore, once you find that you are alive now, as long as there is a nonzero initial probability that persons are repeatable or that the past is finite, you can infer with certainty that either persons are repeatable or the past is finite. If you think the past is infinite, you should infer that persons are repeatable. And if they are repeatable, then they will repeat, given sufficient time. That is how existence is evidence of immortality.

\section{Objections and Replies}

\subsection{The Heat Death of the Universe}

According to the Second Law of Thermodynamics, the universe is fated to constantly increase in entropy until it reaches thermal equilibrium, the state of maximum entropy, in the far future. Thereafter, it will continue in that state forever. In the state of thermal equilibrium, life will be impossible, as will all interesting objects and events. This will prevent your being incarnated ever again, even if persons are in principle repeatable.

In reply, the Second Law of Thermodynamics only records a probabilistic tendency. It is sometimes said that the entropy of a closed system never decreases, and that a system not in thermal equilibrium will always increase in entropy until it reaches thermal equilibrium - but these statements are not strictly true and are in fact refuted by the Poincaré Recurrence 
Theorem. ${ }^{16}$ What is true is that it is extremely improbable that one will observe a spontaneous drop in entropy, on a human time scale. This is why we have never in fact observed this and never expect to. But in an infinite time, "extremely improbable" simply translates to "extremely rare." In the fullness of time, there will eventually be a macroscopic system that spontaneously decreases its entropy by a large amount, even after reaching thermal equilibrium. Eventually, a fluctuation of just the right sort to create a person like you will occur.

That is ignoring the possibility of a systematic mechanism for producing low-entropy states; on some cosmological theories, entropy is systematically reset or diluted at the end of a cycle, or new low-entropy universes are generated. ${ }^{17}$

\subsection{The Physicalist Objection}

Some argue that reincarnation is in principle impossible because it is incompatible with physicalism. ${ }^{18}$ Consider an ordinary material object as an analogy. Suppose that the Mona Lisa is destroyed in a fire at the Louvre. Many years later, someone creates a painting with a nearly identical arrangement of pigments on canvas. We may even imagine that the same atoms are used in the creation of the "new Mona Lisa" as were present in the old painting at the time of the fire. Even so, intuitively, the new painting would merely be a copy. If its creator tries to pass it off as the Mona Lisa, he will be open to charges of fraud.

The point is not specific to artworks but applies to ordinary material objects generally. Once a material object is destroyed, no future object can ever count as the same token object, no matter how similar it may be to the original; any future object can at best be a convincing copy.

Physicalists believe that the world is entirely physical. On this view, it would seem, persons are just physical objects - very complicated and interesting physical objects, indeed, but no more than that. They do not, for example, possess immaterial souls that might migrate to another body when their original body is destroyed. It would therefore seem that, like ordinary material objects, a person, once destroyed, can never be recreated.

In reply, physicalism is not in fact inconsistent with reincarnation. Reincarnation indeed precludes viewing persons as ordinary material objects, given our current conventions for the individuation of ordinary material objects. A physicalist can, however, view persons as temporally gappy spacetime worms. Such gappy spacetime worms are not recognized in our everyday conceptual scheme, but there is nothing absurd and, moreover, nothing particularly non-physicalist about recognizing such objects.

To explain, notice that some material objects may be spatially scattered. For instance, if I own a suit, I might at a particular time have the pants in the closet and the jacket at the dry cleaner. There would thus be a spatial gap between the suit's parts. No one thinks that this requires the suit to possess some special, non-physical component that explains how the two parts can be separated from each other yet be parts of the same suit. Similarly, perhaps the life of a person may be temporally scattered, containing multiple sub-lives that occur in widely separated time periods. This would not require persons to possess a special, non-physical

16 For discussion, see Albert 2000, pp. 71-81.

${ }^{17}$ See footnotes 8 and 9 above.

18 Edwards 1996; Hales 2001. 
component, any more than a suit must have such a component in order to be spatially scattered.

My claim is not that this is in fact the correct view of persons. My claim is that physicalism does not provide a strong reason to reject reincarnation, since the two views can easily be reconciled. If we have good reason to accept physicalism, and if we have independent reason to accept reincarnation, then we should simply adopt a view of persons as gappy spacetime worms.

\subsection{The Dualist Objection}

Having dispatched the physicalist objection, we might face an attack from mind-body dualists, who may object to the notion of there being some purely qualitative conditions for a person to count as you. Cartesian dualists may say that there are no qualitative conditions for a future person to count as you, since any purely qualitative conditions may be satisfied by some future entity and it may fail to be you, simply by not having your particular immaterial soul. Conversely, almost any qualitative conditions may fail to be satisfied by some future conscious being and the being may still be you, simply by having your immaterial soul.

In reply, my case for reincarnation is in fact compatible with Cartesian dualism. Suppose persons have immaterial souls, and that a person lives as long as their soul inhabits some physical body. There remains the question of under what causal conditions your soul comes to inhabit a body. There are Permissive theories, on which the occurrence of certain repeatable conditions causes your soul to be embodied. There are also Restrictive theories, on which your soul can only be embodied once - perhaps, for example, because spatiotemporal continuity with your current body is causally necessary for a future body to be inhabited by your soul. All of the reasoning of section 4 then applies, showing that Restrictive theories are disconfirmed, and Permissive theories confirmed, by your present experience of being alive: Given that the causal conditions for your soul to be embodied are unrepeatable, the probability that you would be alive now is zero. Given that they are repeatable, it is nonzero. Hence, the fact that you are alive now is conclusive evidence that these conditions are repeatable.

\subsection{The Principle of Indifference and A Priori Probabilities}

Some would object to the probabilistic reasoning used above in establishing zero probabilities. For instance, I claim that because there is no a priori reason why one should expect to have been born in the present century rather than the previous century, it is initially at least as likely that one would have been born in the previous century as in this century. Some would object to this sort of reasoning because it is reminiscent of the controversial Principle of Indifference, which holds that when there is no reason for preferring any of a set of alternatives over any other, all the alternatives are equally likely. (This principle is of course meant to apply to epistemic probabilities, not objective chances.)

The Principle of Indifference is controversial because it is possible to divide up the same space of possibilities in different ways, and one can then generate inconsistencies by assigning equal probabilities to "all the alternatives," for different ways of classifying the alternatives. The Principle of Indifference therefore requires a privileged way of dividing up a space of possibilities. Some hold that there is no such privileged division of possibilities, and thus the Principle of Indifference must be rejected. Furthermore, without the Principle of Indifference, 
some would argue, there is no way of assigning a priori probabilities; thus, there can only be probability assessments based on empirical evidence. ${ }^{19}$ Because my arguments above rely upon a priori probabilities, it might be said that these arguments are all unsound.

In other work, I have argued that the paradoxes of the Principle of Indifference can be solved; that there can be no empirical probability assessments without a priori probabilities; that there can be no empirical reasoning in general without a priori probabilities; that the Principle of Indifference in fact underlies the least controversial probability assessments; and that the Principle of Indifference is extremely intuitive. Indeed, the Principle of Indifference may be an analytic truth. However, there is not space to discuss all of these points here. ${ }^{20}$ Here, I shall make two observations apropos of my use of a priori probability assessments.

The first observation to bear in mind is that none of the above arguments require one to hold that all propositions have perfectly precise a priori probabilities. Perhaps only some propositions have a priori probabilities. Perhaps a priori judgments only constrain the range of admissible probability assignments, rather than determining a unique probability distribution. For example, perhaps in certain cases, there are rival, equally admissible partitions of a space of possibilities, and thus multiple rationally acceptable ways of applying the Principle of Indifference, leading to multiple rationally acceptable a priori probability distributions. If so, we might hold that one is rationally required to pick a coherent probability distribution from among those that apply an acceptable interpretation of the Principle of Indifference. Or perhaps one should have indeterminate credences, or credence ranges rather than precise credences.

My second observation is that the sort of probability claims used in my argument are not particularly problematic. For there are not in fact multiple natural ways of partitioning the possibilities in the cases of interest to my argument. In the central case of interest, we assume that a particular person can exist at most once in the history of the universe, and that time is infinite in both directions. We are then to partition the possibilities as to when the person might be born. There is exactly one natural way of doing this: it is to divide the timeline into equal-sized intervals. (Or, if you prefer: apply the Lebesgue measure to the time dimension.) It does not matter that there may be other cases in which we do not know how to decide among rival descriptions of the set of possibilities.

There are admittedly deep problems in this vicinity. There is no way to apply the Principle of Indifference to an unbounded range of possibilities. Thus, suppose you initially know nothing about the universe except that it has some non-negative mass, that is, a mass in the interval $[0, \infty)$. There is no way to give a uniform probability distribution to the possibilities. You cannot give the mass an equal probability of lying in the interval between 0 and 1 grams, between 1 and 2 grams, and so on: If you assign 0 probability to each of these ranges, then you obtain a total probability of 0 ; if you assign a nonzero probability to each range, then you obtain probabilities greater than 1 . You must assign decreasing probabilities for larger possible values. For example, perhaps the mass has a $1 / 2$ probability of lying between 0 and $100 \mathrm{~g}$, a 1/4 probability of lying between 100 and 200 , a $1 / 8$ probability of lying between 200 and 300 , and so on. In that case, the total probability comes to 1 , as desired. There are infinitely many other

${ }^{19}$ van Fraassen 1989, ch. 12; Winsberg 2012, p. 398.

20 See Huemer 2017; 2018, ch. 8. 
probability distributions that give a total probability of 1 ; it is just that none of them are uniform (none assign the same probability for every equal-sized range of possibilities).

Now, one might think that the case of assigning probabilities to your possible time of birth is analogous to assigning a probability distribution to the universe's mass in the preceding example. There is again an unbounded range of possibilities. So one might think that one simply has to use a probability distribution that is skewed toward the present, in the sense that one gives lower probabilities for one's being born at times farther away from the present.

I note two disanalogies between the "time of birth" problem and the "mass of the universe" problem. First, in the latter case, the alternatives are intrinsically, qualitatively different. The situation in which the universe has a mass of 3 grams is intrinsically, qualitatively different from the possible situation in which it has a mass of $10^{56}$ grams. By contrast, however, different times are intrinsically, qualitatively identical. Human systems for representing time have a "zero" point (for example, the time of the birth of Jesus, in the Gregorian Calendar), but there is no natural zero point in objective reality; assuming that time extends infinitely in both directions, no time is any "smaller" or "larger" in itself than any other time. We (presently) have a unique relation to the present, but the present time itself is intrinsically just like every past time, each of which used to be equally present. For this reason, a probability distribution skewed toward "the zero point" makes sense when we are considering possible values of a thing's mass, in a way that it does not make sense when we are considering possible times for some unique event to happen.

The second disanalogy is that we know that the universe had to have exactly one mass in the range from 0 to infinity. It could not have failed to have a value, and it could not have had more than one value. But in the problem where we assign probabilities to your possible birth moments, we do not know that you had to be born at exactly one time. You could have not been born at all, and (on some non-absurd theories) you could have been born at more than one time. Of course, the latter possibility is in dispute; Restrictive theories of persons deny that you could have been born at more than one time. But they cannot deny that you could have failed to be born at all; furthermore, since the Restrictive view is not a priori certain, there is a nonzero prior probability of your having been born at more than one time.

These two disanalogies bear on the probability assignment problem: together, they explain why we need not, and should not, give a probability distribution for your birth time skewed toward the present. We should not do this because there is nothing qualitatively distinct about the present time, and because we have coherent alternatives to privileging the present, such as assigning zero probability to all time intervals, or allowing a person to be born more than once.

\subsection{Can Every Alternative Be Surprising?}

Some would find the argument of section 4.4 suspicious, because it could be applied at any time. The basic claim was that, if persons could exist only once, then it would be surprising that you should exist now, given the infinitely many other centuries in which you could have existed instead. But no matter when you had existed, you could have made the same argument. Any time at which you had found yourself living would have been preceded by an infinite number of other centuries in which you might have existed instead, so any time of birth would have been equally surprising. But, one might think, if every alternative is equally surprising, then really none is surprising. 
Consider an analogy: suppose you flip a coin ten times and record the results. You get heads, tails, heads, heads, tails, tails, tails, tails, tails, heads. ${ }^{21}$ The probability of getting that precise sequence of results is $(1 / 2)^{10}=1 / 1024$. That's pretty improbable! Should you therefore be surprised at the sequence? Presumably not, for that sequence is no less likely than any of the alternatives. Every possible sequence of ten coin-flip results has a probability of 1/1024. And the same thinking applies to the time that you find yourself living. Even if this particular century had zero initial probability of being the one in which you were born, it would not be surprising to be born in this century, if every century had zero initial probability of being the one in which you were born. Or so one might argue.

In reply, the issue is not really one of what observations should count as "surprising." The issue is which observations justify probabilistic inferences about which theories. And what matters to the probabilistic reasoning is not the probability of an observation compared to the otherpossible observations. What matters is the probability of an observation given a particular theory, compared to the probability of that same observation given an alternative theory. In the case at hand, you observe yourself existing in the present century. This is a probability-zero occurrence given the Restrictive view of persons and an infinite past time. It is a nonzeroprobability occurrence given the Permissive view of persons. That is what matters. In other words, letting $\mathrm{H}=$ [Some Permissive theory is correct], $\mathrm{R}=$ [Some Restrictive theory is correct], $\mathrm{L}_{0}=$ [You live in this century], $\mathrm{L}_{-1}=$ [You live in the last century], and so on: What matters is not the comparison between, say, $\mathrm{P}\left(\mathrm{L}_{0} \mid \mathrm{R}\right)$ and $\mathrm{P}\left(\mathrm{L}_{-1} \mid \mathrm{R}\right)$. What matters is the comparison between $\mathrm{P}\left(\mathrm{L}_{0} \mid \mathrm{R}\right)$ and $\mathrm{P}\left(\mathrm{L}_{0} \mid \mathrm{H}\right)$. Because $\mathrm{P}\left(\mathrm{L}_{0} \mid \mathrm{H}\right)>\mathrm{P}\left(\mathrm{L}_{0} \mid \mathrm{R}\right)$, a probabilistic inference favoring $\mathrm{H}$ is justified.

\section{Summary}

In outline, the philosophical argument for reincarnation goes like this:

1. Infinitude of Time: Time is infinite in both directions. (Premise)

2. Infinitude $\rightarrow$ Recurrence: If the future is infinite, then every repeatable condition that obtains has obtained before, and will obtain again, infinitely many times. (Premise)

3. Recurrence $\rightarrow$ Reincarnation: If every repeatable condition that obtains will obtain again infinitely many times, then you will be reincarnated infinitely many times. For:

a. The probability of your being alive now, given an infinite past and given that the conditions required for you to be incarnated are unrepeatable, is zero. (Premise)

b. You are alive now. (Premise)

c. The past is infinite. (From 1)

d. Therefore, the conditions required for you to be incarnated are repeatable. (From 3a, 3b, 3c)

e. Therefore, if every repeatable condition that obtains will obtain again infinitely many times, then you will be reincarnated infinitely many times. (From 3d)

4. Reincarnation: Therefore, you will be reincarnated, infinitely many times. (From 1, 2, 3)

21 This is an actual random sequence generated by my computer. 
Premise $3 \mathrm{a}$ is the most likely target of philosophical suspicion; this premise embodies the most unusual type of thinking involved in the argument. That thinking is nevertheless correct. If you were to live at most once in the history of the universe, we cannot justify assigning any probability greater than zero, initially, to your living at the present time, given an infinite number of past centuries in which you could have been born instead. The best explanation for your otherwise surprising present existence is that you are repeatable, so that your living at an earlier time would not have prevented you from living now - in which case, your present life also will not prevent you from living any number of future lives.

\section{References}

Albert, David Z. 2000. Time and Chance. Cambridge, MA; Harvard University Press.

Almeder, Robert. 1992. Death and Personal Survival: The Evidence for Life After Death. Lanham, Md.: Rowman \& Littlefield.

Ashtekar, Abhay. 2009. "Loop Quantum Cosmology: An Overview," General Relativity and Gravitation 41: 707-41.

Baum, Lauris and Paul H. Frampton. 2007. "Turnaround in Cyclic Cosmology," Physical Review Letters 98: 071301(4).

Carroll, Sean. 2010. From Eternity to Here: The Quest for the Ultimate Theory of Time. New York: Dutton.

Chen, Eddy K. 2017. "Being Indifferent Towards the Past: Some Puzzles about De Se Probabilities and Time's Arrow," manuscript.

Craig, William Lane. 1991. "The Existence of God and the Beginning of the Universe," Truth: A Journal of Modern Thought 3: 85-96. Available at <http://www.leaderu.com/truth/3truth11.html>, accessed October 20, 2018.

Craig, William Lane. 2001. Time and Eternity: Exploring God's Relationship to Time. Wheaton, IL: Crossway Books.

Davies, Paul. 1984. God and the New Physics. New York: Simon \& Schuster.

Edwards, Paul. 1996. Reincarnation: A Critical Examination. Amherst, N.Y.: Prometheus.

Hacking, Ian. 1987. “The Inverse Gambler's Fallacy: The Argument from Design. The Anthropic Principle Applied to Wheeler Universes," Mind 96: 331-40.

Hales, Steven D. 2001. "Evidence and the Afterlife," Philosophia 28: 335-46.

Hempel, Carl. 1960. "Inductive Inconsistencies," Synthese 12: 439-69.

Hirsch, Eli. 2005. "Physical-Object Ontology, Verbal Disputes, and Common Sense," Philosophy and Phenomenological Research 70: 67-97.

Huemer, Michael. 2016. Approaching Infinity. New York: Palgrave Macmillan.

Huemer, Michael. 2017. "There Is No Pure Empirical Reasoning," Philosophy and Phenomenological Research 95: 592-613.

Huemer, Michael. 2018. Paradox Lost. New York: Palgrave Macmillan.

Jenkins, Scott. 2012. "Time and Personal Identity in Nietzsche's Theory of Eternal Recurrence," Philosophy Compass 7: 208-17.

Mithani, Audrey and Alexander Vilenkin. 2012. "Did the Universe Have a Beginning?”, arXiv:1204.4658. 
Nietzsche, Friedrich. 2001. The Gay Science, ed. Bernard Williams, tr. Josefine Nauckhoff. Cambridge: Cambridge University Press.

Nietzsche, Friedrich. 2003. Writings from the Late Notebooks, ed. Rüdiger Bittner, tr. Kate Sturge. Cambridge: Cambridge University Press.

Penrose, Roger. 2010. Cycles of Time: An Extraordinary New View of the Universe. New York: Random House.

Seneca, Lucius Annaeus. 2010. Selected Letters, tr. Elaine Fantham. Oxford: Oxford University Press.

Steinhardt, Paul J. and Neil Turok. 2005. "The Cyclic Model Simplified," New Astronomy Reviews 49 (2005) 43-57.

Susskind, Leonard. 2006. The Cosmic Landscape. New York: Little, Brown and Co.

van Fraassen, Bas. 1989. Laws and Symmetry. Oxford: Clarendon.

van Inwagen, Peter. 1978. "The Possibility of Resurrection," International Journal for the Philosophy of Religion 9: 114-21.

Wallace, David. 2015. "Recurrence Theorems: A Unified Account," Journal of Mathematical Physics 56: 022105(11).

Winsberg, Eric. 2012. "Bumps on the Road to Here (from Eternity)," Entropy 14: 390-406. 